\title{
ASPECTOS BIOLÓGICOS E FISIOLÓGICOS DO ENVELHECIMENTO HUMANO E SUAS IMPLICAÇÕES NA SAÚDE DO IDOSO
}

\author{
Francisco Luiz de Marchi Netto*
}

\section{RESUMO}

Trata o presente texto de uma discussão acerca dos principais aspectos biológicos e fisiológicos do corpo humano, vivenciados a partir da idade adulta e principalmente na terceira idade, os quais apresentam-se com maior fragilidade no que se refere aos padrões normais obtidos durante as fases infantil e adultas do indivíduo. O tema desperta a ênfase para as práticas regulares das atividades físicas (exercícios, esportes, danças, lutas, etc.) como uma das formas saudáveis para contrapor e atenuar as causas e os efeitos do envelhecimento corporal, que acomete todas as pessoas na terceira idade.

PALAVRAS-CHAVE: Envelhecimento Biológico-Capacidades Funcionais - Exercícios - Terceira Idade.

\section{INTRODUÇÃO}

A era contemporânea caracteriza-se por diversas transformações: históricas, filosóficas, econômicas, políticas e sociais, as quais deixam um legado importante para a humanidade. E um dos fenômenos sociais que mais tem se destacado e demarcado seu espaço é o aumento acelerado da população de idosos, que ocorre praticamente em todo o mundo.

A população brasileira, que até bem pouco tempo (décadas de 1970 a 1980) era considerada jovem, conta hoje com cerca de $23 \%$ de seus indivíduos com idade superior a 60 anos, o que os enquadra na chamada Terceira Idade. Segundo apontam os números do censo do IBGE (2001), os cidadãos brasileiros que já se encontram acima dos 60 anos somam aproximadamente 14,3 milhões de habitantes. Esta mesma fonte estatística projeta para o ano 2025 um crescimento da população de idosos que colocará o Brasil como o sexto país do mundo no ranking dos países com o maior número de idosos entre os seus habitantes (CORAZZA, 2001).

\footnotetext{
* Especialista e Professor da Faculdade de Educação Física da UFG
} 
Idade e envelhecimento da população (OMS)

IDADE MÉDIA - 45 aos 59 anos

PESSOAS IDOSAS - 60 aos 74 anos

VELHICE - 75 aos 89 anos

GRANDE VELHICE - a partir dos 90 anos

Fonte: Vieira (1996).

\section{População de idosos no Brasil (IBGE, 2001)}

Assim, observa-se que especialmente nas últimas três décadas, uma parte bastante significativa da população brasileira tem atingido e permanecido por mais tempo nas faixas etárias da Terceira Idade.

$$
\begin{aligned}
& 950-4,2 \% \\
& 1994-7,7 \%=10,5 \text { milhões } \\
& 2000-8,3 \%=14,3 \text { milhões } \\
& 2025-15 \%=35 \text { milhões }
\end{aligned}
$$

Segundo aponta a Organização Mundial de Saúde no ano de 2001 o Brasil tinha uma população total e de idosos de acordo com o

\begin{tabular}{|c|c|c|}
\hline Regiões & População & $\begin{array}{c}\text { Total População de } \\
\text { Idosos }\end{array}$ \\
\hline Norte & 6.556 .548 & 362.901 \\
\hline Nordeste & 45.448 .490 & 3.826 .662 \\
\hline Centro-Oeste & 1.910 .443 & 738.261 \\
\hline Sudeste & 68.280 .153 & 6.518 .218 \\
\hline Sul & 23.932 .379 & 2.046 .138 \\
\hline
\end{tabular}
quadro abaixo:

Quadro 1 - Distribuição da população nas 5 (cinco) regiões do Brasil.

Fonte: Corazza, 2001. 
Um dos aspectos importantes que mais vem sendo discutido pelos estudiosos do meio acadêmico que pesquisam sobre este tema, é que esta fase de vida não é alcançada de uma forma satisfatória sem que se façam presentes os surgimentos de problemas, quer sejam de ordem orgânica ou psicológica.

Neste sentido é que abordaremos neste artigo, alguns dos aspectos intervenientes das funções biológicas e psicológicas de pessoas da terceira idade e suas implicações na saúde, a fim de que possamos refletir acerca desta prática voltada a esta parcela cada vez crescente da população brasileira.

Os estudos apresentados por Faria Júnior (1997) denunciam as precárias condições orgânicas e de saúde da população de idosos, especialmente aqueles mais desprovidos de condições financeiras, residentes em abrigos e asilos existentes na cidade do Rio de Janeiro. Os estudos apontam para elevado índice de deficiência das capacidades físicas e motoras dessa população, devido à precariedade de todo o sistema de atendimento a essas pessoas. Todavia, se olharmos bem ao nosso redor, constataremos que essa realidade não é diferente nas demais cidades brasileiras, ainda nos dias de hoje. Outros estudos da mesma natureza, nos chamam a atenção para que observemos a forma como a nossa sociedade, pautada nos princípios capitalista e de produtividade, discrimina e segrega os idosos menos favorecidos social e economicamente.

Uma vez que os avanços da ciência e da medicina contribuem para o prolongamento da vida das pessoas, faz-se necessário repensar e colocar em prática formas mais humanizadoras de convivência com as pessoas da Terceira Idade, tanto internamente nas famílias como nos serviços de atendimento e população em geral.

Na concepção de Vieira (1996) e Lopes (2000), os processos de envelhecimento se iniciam desde a concepção, sendo então a velhice definida como um processo dinâmico e progressivo no qual ocorrem modificações, tanto morfológicas, funcionais e bioquímicas, como psicológicas, que determinam a progressiva perda das capacidades de adaptação do indivíduo ao meio ambiente, ocasionando maior vulnerabilidade e maior incidência de processos patológicos. Sociólogos e psicólogos chamam a atenção para o fato de que, além das alterações biológicas, podem ser observados processos de desenvolvimento social 
e psicológicos alterados em algumas das suas funções, como também problemas de integração e adaptação social do indivíduo.

Okuma (1998), acrescenta que a velhice não é definível por simples cronologia, e sim pelas condições físicas, funcionais, mentais e de saúde do indivíduo, sugerindo que o processo de envelhecimento é pessoal e diferenciado. Nessa perspectiva, a autora considera que o envelhecimento humano constitui um padrão de modificações e não um processo unilateral, mas sim, a soma de vários processos entre si, os quais envolvem aspectos biopsicossociais.

Portanto, na velhice como em qualquer outra idade, há pessoas sãs e pessoas doentes. A verdade é que muitas das enfermidades, supostamente próprias da velhice, e que já existiam antes da chegada desta faixa etária, apenas se manifestavam com menor intensidade, porém agora, aceleram o seu curso. Isto não exclui o fato de que com o passar dos anos, processe-se no organismo mudanças naturais que constituam uma velhice sã e normal.

Com a chegada da velhice, as alterações anatômicas são principalmente as mais visíveis e manifestam-se em primeiro lugar. A pele que resseca, tornando-se mais quebradiça e pálida, perdendo o brilho natural da jovialidade. Os cabelos que embranquecem e caem com maior frequiência e facilidade não são mais naturalmente substituídos, principalmente nos homens. O enfraquecimento do tônus muscular e da constituição óssea leva a mudanças na postura do tronco e das pernas, acentuando ainda mais as curvaturas da coluna torácica e lombar. As articulações tornam-se mais endurecidas, reduzindo assim a extensão dos movimentos e produzindo alterações no equilíbrio e na marcha. Nas vísceras, produz-se uma alteração causada pelos elementos glandulares do tecido conjuntivo e certa atrofia secundária, como a perda de peso. Quanto ao sistema cardiovascular, é próprio das fases adiantadas da velhice a dilatação aórtica e a hipertrofia e dilatação do ventrículo esquerdo do coração, associados a um ligeiro aumento da pressão arterial.

$\mathrm{Na}$ parte fisiológica, as alterações, na maioria das vezes, podem ser observadas pela lentidão do pulso, do ritmo respiratório, da digestão e assimilação dos alimentos. Porém, acima de tudo, o próprio indivíduo sente a decadência de sua capacidade de satisfação sexual. O organismo torna-se cada vez mais difícil para ambos os sexos, contudo, a atividade sexual não desaparece, apenas torna-se menos intensa e freqüente. 
Todavia, a manutenção pelo interesse sexual após os 60 anos de idade é um dos aspectos considerados como um sinal de manutenção das boas condições de saúde. Estudos comprovam que a atividade sexual alivia as artrites, aumenta a produção de cortisona das glândulas supra-renais e contribui igualmente para o equilíbrio psíquico. É falso acreditar que as pessoas que sofrem problemas coronarianos correm risco de uma crise cardíaca durante a relação sexual.

Uma das facetas do problema biológico é o medo da morte. Todos nós, uns mais outros menos, tememos a enfermidade e a invalidez. Esse medo não se justifica apenas pelo agravamento dos sistemas funcionais, mas pela perspectiva de que este seja o prenúncio do mal que leva à morte.

Conforme pode-se constatar, verificamos descritas no quadro a seguir algumas das principais funções fisiológicas do corpo humano, bem como as mudanças biológicas e estruturais que diminuem gradativamente e naturalmente as suas funções, tendo em vista o envelhecimento dos órgãos e tecidos, os quais atingiram o ápice de seu funcionamento durante a fase adulta.

Quadro 2- Mudanças biológicas e funcionais entre idades de 30 a 80 anos.

\begin{tabular}{|c|c|}
\hline FUNÇÃO BIOLÓGICA & MUDANÇAS \\
\hline $\begin{array}{l}\text { Capacidade de Trabalho \% } \\
\text { Débito Cardiáco } \\
\text { Freq. Card. Máx.(bat./Min.) } \\
\text { Pressão Sanguínea (mm/hg) } \\
\text { PAS } \\
\text { PAD } \\
\text { Ventilação } \\
\text { Capacidade } \\
\text { Volume Residual } \\
\text { Metabolismo(\%) } \\
\text { Musculatura } \\
\text { Massa Muscular } \\
\text { Forca de Preensão }\end{array}$ & $\begin{array}{l}\text { Dim*25 - } 30 \\
\text { Dim* } 30 \\
\text { Dim* } 24 \\
\text { Aum** 10 - 40 } \\
\text { Aum** } \mathbf{0 5}-\mathbf{1 0} \\
\text { Dim* } 40-50 \\
\text { Dim** } 30-40\end{array}$ \\
\hline
\end{tabular}




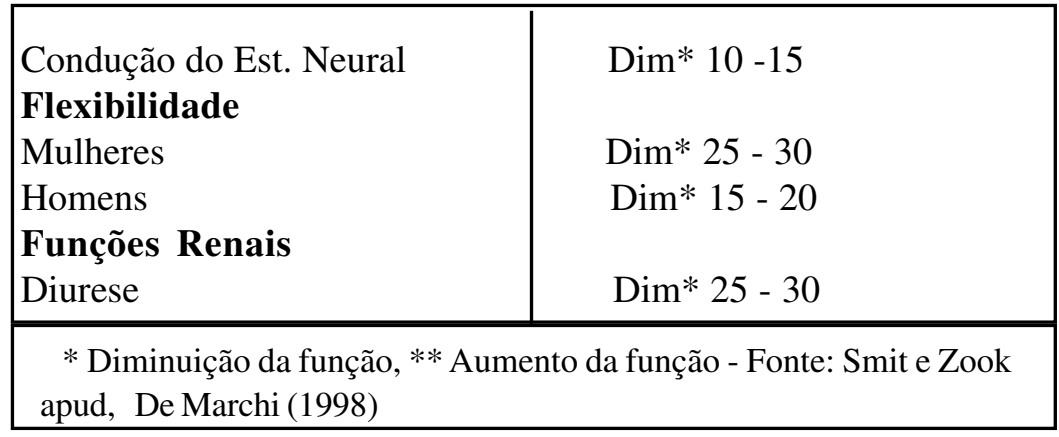

Fonte: Smit e Zook apud De Marchi (1998)

Pode-se perceber que os autores apresentam valores médios de perdas em percentuais de cada uma das variáveis pesquisadas, depois de um estudo longitudinal com pessoas normais, das faixas etárias de 30 a 70 anos. Outros estudos realizados e divulgados no meio científico sugerem que essas perdas podem ser minimizadas com a prática regular de atividades físicas, as quais atuam na manutenção de índices normais e/ou até mais elevados, como é o caso de indivíduos que participam de atividades esportivas nas categorias masters ${ }^{l}$.

A seguir, verificamos algumas das modificações e alterações celulares, principalmente do tecido muscular devido ao avanço da idade, após a fase adulta.

Quadro 3 - Alterações das células musculares na terceira idade.

\begin{tabular}{|l|l|}
\hline Funções fisiológicas & $\begin{array}{l}\text { Alterações morfológicas e } \\
\text { bioquímicas }\end{array}$ \\
\hline Diminuição da força & $\begin{array}{l}\text { Diminuição de fibras na contração } \\
\text { muscular } \\
\text { Atrofia das fibras do tipo II. } \\
\text { Irregularidade na estrutura dos } \\
\text { sarcômeros. }\end{array}$ \\
& \\
\hline
\end{tabular}




\begin{tabular}{|c|c|}
\hline & $\begin{array}{l}\text { Alteração das enzimas ATPase } \\
\text { da miosina }\end{array}$ \\
\hline $\begin{array}{l}\text { Contração muscular mais } \\
\text { lenta }\end{array}$ & $\begin{array}{l}\text { Alterações funcionais do } \\
\text { retículo do sarco-plasma; } \\
\text { Atrofia das fibras do tipo II } \\
\text { Diminuição da excitabilidade } \\
\text { neural } \\
\text { Mudanças nas bombas iônicas } \\
\text { do sarcolema } \\
\text { Desidratação e níveis mais } \\
\text { baixos de potássio } \\
\text { Aumento do tecido conjuntivo } \\
\text { no interior das fibras }\end{array}$ \\
\hline
\end{tabular}

Fonte: Matsudo e Matsudo (2001)

Também é importante salientar que devido às influências multifatoriais do envelhecimento, ocorrem no idoso, alterações nos reflexos de proteção e no controle do equilíbrio, prejudicando-se assim a mobilidade corporal. As funções identificadas no quadro 3 são altamente suscetíveis ao efeito da falta de atividades físicas e motoras. Portanto, os indivíduos da terceira idade que buscam fazer uso da prática regular no campo da cultura corporal, propiciam ao organismo uma diminuição ou desaceleração da velocidade do espiral descendente, a qual implica em suscetibilidades diante dos problemas que envolvem a mobilidade.

Assim, pode-se inferir que os aspectos ligados à mobilidade estão diretamente voltados à saúde das estruturas ósseas, que na terceira idade apresenta-se de uma forma mais comprometida do que na fase adulta, especialmente àquelas pessoas que têm como hábito o fumo e a ingestão elevada de cafeína e álcool, associados a uma alimentação pobre em cálcio. Os problemas de osteoporose, ao contrário do que se pensa, não estão ligados apenas ao risco de fraturas, mas também implicam na diminuição da mobilidade articular, em função do desgaste ósseo das articulações (encaixes ósseos).

As fraturas são o último fato a ser acometido aos Ossos com 
desgaste, ocorrendo quando estes forem submetidos a uma força de pressão demasiadamente mais elevada do que a sua própria estrutura poderia suportar. As ocorrências mais comuns de fraturas na terceira idade são aquelas ligadas ao desequilíbrio e às quedas, provocando fraturas no colo do fêmur, quadril ou vértebras lombares, geralmente levando o indivíduo a ficar imobilizado por muito tempo em leitos hospitalares, e esse fato tem ocasionado a morte a uma série de pessoas da terceira idade.

Veja a seguir algumas das alterações dessas capacidades que envolvem a mobilidade corporal em função do avanço da idade.

Quadro 4 - Alterações que implicam na diminuição da mobilidade na terceira idade.

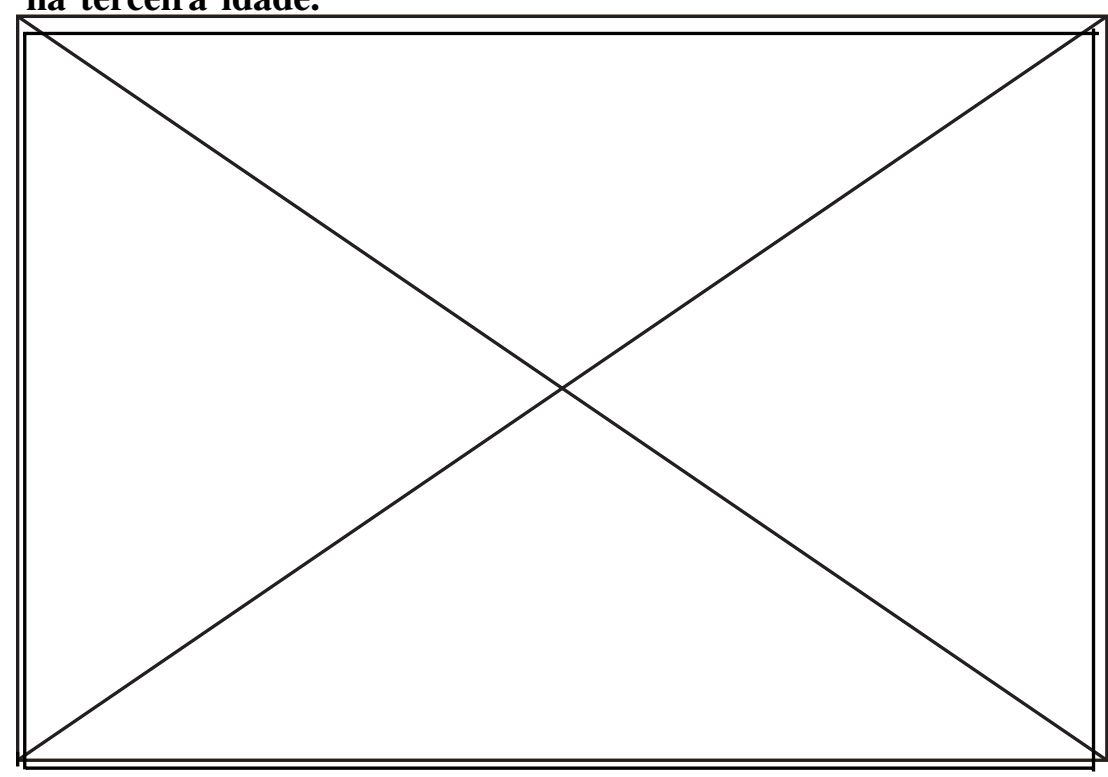

Fonte: De Marchi (1998)

\section{CONCLUSÃO}

Embora estes quadros apresentem dados nada agradáveis à primeira vista, não se pode avaliá-los de forma preconceituosa e pessimista. $\mathrm{O}$ fato real e natural é que todo ser vivo envelhece no seu aspecto orgânico e biológico, muito embora nem todo ser vivo envelheça 
na mesma proporção, nos sentidos psicológico, espiritual e humano. Assim, as recomendações para a orientação e o acompanhamento da prática de atividades físicas leves e moderadas são importantes por estimular os idosos a se envolverem em programas de exercícios e atividades físicas, assegurando-se, deste modo, a manutenção e recuperação das funções vitais do organismo, as quais auxiliam também as funções neurocerebrais e músculo-esqueléticas.

Portanto, as funções intelectuais nas pessoas da terceira idade, necessitam também de estímulos, a fim de que se mantenham em plenas condições favoráveis de atividades, num trabalho contínuo e cotidiano, como por exemplo: a leitura, o estudo, o raciocínio, a reflexão, a meditação, entre outros.

Biological and physiological aspects of aging and its implications in the health of the elderly

\section{ABSTRACT}

This text deals with a discussion on the main biological and physiological aspects of the human body that are experienced in adulthood, especially among the elderly, who are more fragile regarding the regular standards obtained during childhood and earlier adulthood. This text emphasizes regular physical activity (exercise, sports, dance, martial arts, etc) as one of the healthy ways to oppose and ease the causes and effects of bodily aging that affect the elderly.

KEY WORDS: Biological aging - Ffunctional capacities - Eexercise - Elderly,Tthird age

Aspectos biologicos y fisiologicos del envejecimiento humano u sus implicaciones en la saude del idoso

\section{RESUMEN}

Trata el presente texto de una discusión acerca de los principales aspectos biológicos y fisiológicos del cuerpo humano, vivenciados a partir de la edad adulta y principalmente en la tercera edad, los cuales se presentan con mayor fragilidad en lo que se refiere a los modelos normales obtenidos durante las fases infantil y adultas del individuo. El tema despierta el énfasis para las prácticas regulares de las actividades físicas (ejercicios, deportes, danzas, luchas, etc.) como una de las formas saludables para contraponer y atenuar las causas y los efectos del envejecimiento corporal, que acomete todas las personas en la tercera edad.

PALABRAS CLAVES: Envejecimiento Biológico - Capacidades Funcionales Ejercicios - Tercera Edad 


\section{NOTAS}

${ }^{1}$ Denominação atribuída às categorias esportivas praticadas por pessoas com idades igual ou superior a 40 anos

\section{REFERÊNCIAS}

CORAZZA, M. A. Terceira Idade e Atividade Física. São Paulo: Phorte, 2001.

DE MARCHI, F.L. As capacidades físicas e funcionais na terceira idade. Texto didático, Faculdade de Educação Física - UFG, 1998.

FARIA JÚNIOR, A. Atividade Física para a terceira idade. Brasília: SESI -INDESP, 1997.

IBGE - Instituto Brasileiro de Geografia e Estatística - Brasília, DF: Censo Demográfico Brasileiro, 2001

LOPES, A. Os desafios da gerontologia no Brasil. Campinas - SP: Alínea, 2000.

MATSUDO, S. M. R \& MATSUDO, V. K. A atividade física e o idoso. São Paulo: Manole, 2001.

OKUMA, S. S. O idoso e a atividade física. Campinas, São Paulo: Papirus, 1998.

VIEIRA, E. B. Manual de gerontologia. Rio de Janeiro: Revinter, 1996. Recebido: novembro de 2003 Aprovado: dezembro de 2003

Endereço para correspondência Prof. Francisco Luiz De Marchi Netto - FEF/UFG e-mail:demarchi@fef.ufg.br Av. Pedro Paulo Sousa, 1870, Qd3, 1t.4, Bloco 3b Apto.08 - Goiânia 2 CEP - 74.000-000 Goiânia - GO 